\title{
Desafios da Sustentabilidade Educacional e as contribuições da Tecnologia Cloud Computing
}

\author{
Stela C. Bertholo Piconez ${ }^{1}$, Adalberto Bosco Pereira², Josete M. Zimmer ${ }^{3}$, \\ Oscar Luiz Piconez Filho ${ }^{3}$ \\ ${ }^{1}$ Faculdade de Educação - USP \\ ${ }^{2}$ Instituto de Matemática - USP \\ ${ }^{3}$ Grupo Alpha de Pesquisa - FEUSP/CNPq \\ stela.piconez@gmail.com; adalbertoboscolgmail.com; \\ josete.zimmer@gmail.com; oscake@gmail.com
}

\begin{abstract}
This article aims future trends in Education with technologies with the purpose of expanding the idea of Educational Sustainability supported by the technological innovation of Cloud Computing. Likewise, it aims to relate the two elements present, their challenges and their mutual contributions to address the strategic possibilities of interaction based on complex, creative, transdisciplinary and transcultural epistemology. It aims to understand the formal, informal and corporate spaces and their relevant social functions as well contributions to the empowerment of global citizenship. Education with technologies, at all levels of education, can foster interactivity and interaction regarding the possibilities of educational intentionality. It defends a new communication posture, based on the authorship of the user, on the logic of interactivity and constantly (re) signified.
\end{abstract}

Resumo. Este artigo discute futuras tendências de Educação com tecnologias que favoreçam o debate sobre as questões de Sustentabilidade Educacional e o suporte da tecnologia Cloud Computing. Da mesma forma, visa relacionar os dois elementos presentes, seus desafios e contribuições mútuas para abordar as possibilidades estratégicas de interação fundamentada por epistemologia complexa, criativa, transdisciplinar e transcultural. Objetiva o entendimento sobre os espaços formais, informais e corporativos e suas funções sociais relevantes como contribuição ao fortalecimento de cidadania global. Educação com tecnologias, em todos níveis elou modalidades de ensino, pode favorecer a interatividade e a interação quanto às possibilidades de intencionalidade educativa. Defende uma nova postura comunicacional, alicerçada na autoria do usuário, na lógica da interatividade e constantemente (re) significadas.

\section{Introdução}

Este artigo destaca os desafios e as contribuições da Cloud Computing vislumbrando e apontando temas desafiadores e interdisciplinares, com enfoque basilar na área de Informática na Educação, especificamente na Sustentabilidade Educacional. Tem por objetivo discutir os temas da Informática na Educação que possuam sobretudo aspectos desafiadores na área. Basicamente se orientam pelas questões que seguem: 


\section{Que desafios a Educação tem e que a Computação pode ajudar a resolver?}

\section{Como conferir Sustentabilidade Educacional com apoio da computação?}

Para aclaramento dos termos, Sustentabilidade Educacional é entendida como o desenvolvimento de uma visão integrada dos saberes que contemplem o desenvolvimento da aprendizagem docente e discente na direção da inclusão de questões éticas, epistemológicas e pedagógicas necessárias ao processo de construção e compartilhamento de conhecimentos. Pretende desafiar paradigmas, questionar e provocar uma evolução no panorama atual na área educacional além de identificar potenciais dificuldades atuais e vindouras inerentes ao desafio de apropriação das tecnologias tanto na educação escolar formal, quanto nos espaços informais e coorporativos.

Cloud Computing (computação nas nuvens) como inovação em computação na última década, possui potencial na recolha, organização e exploração dos dados sobre diversas perspectivas. Exige um pouco de investimento, mas todos concordam que o seu desenvolvimento e implementação proporcionarão enormes vantagens competitivas às organizações como um todo (Tran, et al., 2013; Zanetti et al., 2016). Ela é compreendida como uma tendência após a internet, a partir de 1995, de deixar de ser exclusivamente acadêmica, comercial e/ou industrial para ser usada em escala mais global (Zuffo et. al., 2013; Ekanayake, and Fox,G., 2010; NIST 2013).

Especificamente em relação à educação escolar, a padronização de equipamentos de ensino multimídia pode permitir total acesso e viabilizar para estudantes e professores, em diferentes áreas de conhecimento, facilidade de acesso e interação com a tecnologia web em tempo real. A internet apoia textos compartilhados de comunicação entre colegas de sala e amigos nas redes sociais sob diferentes perspectivas de comunicação, informação e criação. Salas de aula multimídia, personal computers, equipamentos móveis favorecem outros métodos de aprendizagem para diferentes demandas de outros locais e encontram no "cloud services", via web (rede de informações pela internet (rede de computadores), a mobilidade, cooperação, o arquivamento de dados, a elasticidade e a escalabilidade (Righi, R, 2013) e facilidade de acesso. Fortalecem, ainda, o desenvolvimento de competências escolares (conceituais, procedimentais e atitudinais), de modo que todos os estudantes possam receber a mesma informação independente de seus conhecimentos tácitos (anteriores) e/ou das áreas de conhecimento desenvolvidas na escola e aprendam com qualidade e igualdade (Piconez, 2004; 2013).

A internet apoia textos compartilhados de comunicação entre colegas de sala e amigos nas redes sociais sob diferentes perspectivas de comunicação, informação e criação. Salas de aula multimídia, personal computers, equipamentos móveis favorecem outras abordagens de ensino e aprendizagem (pedagogia, andragogia, heutagogia) para diferentes demandas. Encontra no "cloud services", a mobilidade, cooperação, o arquivamento de dados, a escalabilidade e facilidade de acesso.

Um dos desafios é que a computação em nuvem já existe na escola, nos espaços informais e coorporativos se pensarmos o acesso que as pessoas já têm por meio das redes sociais, dos provedores de busca, dispositivos móveis (BYOD) e da enorme quantidade de informação armazenada. A computação em nuvem utiliza as grandes quantidades de informação geradas, procura organizá-las e utilizá-las como meio de suporte consistente, viável e capaz de assumir como pressuposto basilar a tomada de decisão para resolução de problemas. No cenário atual da educação no Brasil existe precariedade na infraestrutura tecnológica escolar, na formação docente e na continuidade de uso de abordagem tradicional de ensino (perspectiva mnemônica). É relevante conhecer noções de programação e de raciocínio computacional. Todos na escola precisam ter uma cultura 
digital (digital literacy), isto significa, saber acessar e encontrar informações, apps, competência cada vez mais exigida pelo mundo contemporâneo (Grassian e Kaplpwitz, 2001). E ainda, não podemos esquecer da familiaridade com a nuvem e a cibersegurança (informational literacy).

Tal como referido acima, o objetivo deste artigo destina-se à reflexão para profissionais que trabalham ou possam trabalhar agora e em um futuro próximo, encontrando na tecnologia cloud computing e em sua arquitetura, o suporte para as soluções, desafios e contribuições presentes na Sociedade do Conhecimento. Por outro lado, visa igualmente abordar temas relacionados a desafios já identificados pela comunidade acadêmica e escolar, tais como: Acesso Participativo e Universal do Cidadão ao mesmo conhecimento e de mesma qualidade pela abertura à todas as escolas de acesso ao cloud computing, autoria de sistemas educacionais, evolução de sistemas educacionais, gestão de grandes volumes de dados de desenvolvimento de tecnologias educacionais de alta qualidade, entre outros. As políticas e estratégias existentes no desenvolvimento da cultura digital e informacional devem estar amparadas em um conceito que harmonize e inclua toda e qualquer acesso pela internet, que abrange os mesmos conhecimentos e qualidade, habilidades e atitudes que permitam aos cidadãos compreender o papel social das mídias e de outros provedores nas sociedades democráticas que requerem liberdade de expressão, diálogo intercultural e possibilidades de criação de conhecimento colaborativo.

\section{A Sustentabilidade Educacional e o Cloud Computing: desafios}

O fenômeno educativo é complexo e multidimensional. A Sustentabilidade Educacional requer a provisão de espaços permanentes de estudo e conhecimento das tecnologias para que ocorra uma adaptação tanto de estudantes quanto dos docentes na direção da sensibilidade do contexto que vislumbra novos modos de ensinar e novos modos de aprender. Para ilustrar, se o projeto da escola quer utilizar os games digitais para desenvolver competências lógico-algébricas e mesmo de pensamento computacional com os estudantes, os docentes precisam jogar, aprender a articular as competências e habilidades desenvolvidas aos conteúdos específicos da matemática como projeto integrador (De Paula et al., 2014; Pereira, A. B. e Piconez, S.C.B, 2016; Barr, V. e Stephenson, C. 2011; Brennan, K. e Resnick, M. (2012; Mestre, P. et al. 2015).

Se a modalidade por smartphones é real, a mobilidade tecnológico-educacional tem como pressuposto acesso irrestrito como forma de independência dos dispositivos de acesso aos conteúdos. Pretende-se com o acesso à computação nas nuvens, que professores e alunos acessem e explorem os dados, de forma dinâmica e interativa.

A função social da escola é ser sustentável com a estrutura que aí está; mudanças levam dez anos para fazer efeito na Educação. Então, pela tecnologia de Cloud Computing os desafios para computação consistem em formar docentes e discentes em técnicas de computação existentes na programação de jogos, edição de vídeos com Moviemaker, Animoto, iMovie, uso da internet das coisas, uso da realidade aumentada, das narrativas digitais utilizando Instagram; redes de comunidades de prática para intercâmbio de línguas, contextos, cenários etc como grupos em Whatsapp, FaceBook-Messenger que podem ajudar a resolver o problema de propiciar a todos a mesma qualidade e acesso aos mesmos conteúdos existentes, objeto de estudo no currículo escolar.

Casell (2011) destaca que a Ciência da Computação (CC) é uma área do conhecimento de caráter universal. Favorece um leque de possibilidades para a realização de conexões interdisciplinares, tanto para a sua evolução enquanto ciência, quanto para a sua aplicação na resolução de problemas mais complexos. Esses problemas podem ser 
explorados por meio da interdisciplinaridade, que é apenas uma postura metodológica e que as escolas não conseguem viabilizá-la por questões administrativas-burocráticas em sua organização e gestão.

No campo da Educação Escolar, há tentativas de articulações conceituais presentes nos componentes curriculares obrigatórios, como por exemplo, dos games digitais, tão apreciados pelos estudantes com imensa possibilidade e articulação com a linguagem abstracional matemática e de seus algoritmos.

De acordo com Blikstein (2010a, 2010b) a escola faz "adestramento digital" Pior, estamos ensinando nossos alunos que a tecnologia serve para recombinar informações já existentes, e não para criar conhecimento novo. E o conhecimento novo não está na internet, facilmente encontrável em um mecanismo de busca com meia dúzia de palavraschave. Ele está por ser descoberto. Ainda segundo o autor, não se trata de saber navegar na internet, enviar e-mail, publicar um blog, ou operar um processador de texto. Raciocínio computacional é saber usar o computador como um instrumento de aumento do poder cognitivo e operacional humano - em outras palavras, usar computadores, e redes de computadores, para aumentar nossa produtividade, inventividade e criatividade.

\section{Como vencer o Desafio?}

Vivemos em simbiose com os artefatos tecnológicos, ou seja, os seres humanos estão conectados simultaneamente às tecnologias. Na escola não é diferente. Para Pierre Lévy é o uso das tecnologias que nos faz terrivelmente humanos. Os estudantes de hoje consideram as tecnologias como artefatos absolutamente normais e cotidianos. Se a escola proibir os estudantes de usar as tecnologias (para os professores algo separado do mundo), na cabeça dos estudantes, estamos proibindo-os de viver. Nossas escolas não sabem como ensinar com tecnologias. Só que projetar estudos com pesquisa na internet tem duas questões importantes de sustentabilidade educacional, a saber: a riqueza de informações não desenvolve de per si a capacidade analítica, a articulação da atenção, a motivação e a interpretação das ideias assim como produção de conhecimentos novos e compartilhados.

A habilidade de transformar teorias e hipóteses em modelos e programas de computador, executá-los, depurá-los, e utilizá-los para redesenhar processos produtivos, realizar pesquisas científicas ou mesmo otimizar rotinas pessoais, é uma das mais importantes habilidades para os cidadãos do século XXI. E, curiosamente, é uma habilidade que nos faz mais humano. Precisamos urgentemente redirecionar nossos esforços e recursos para ensinar nossos estudantes a compreender esse interessante paradoxo: o raciocínio computacional nos torna cada vez mais dependente e, ao mesmo tempo, diferentes dos computadores. Entender como podemos ser mais produtivos e criativos - mesmo sendo mais dependentes - é o maior desafio dos educadores que querem repensar a tecnologia na sala de aula (Blikstein, 2008).

O quadro 1 apresenta as dimensões de contextos existentes na Sociedade do Conhecimento com Educação Formal, Informal e Coorporativa; no Contexto Educacional e Sustentabilidade. Para formulação de algumas ideias para as diferentes áreas de enfoque, foram elencadas metas estratégicas de implementação de diferentes ações que possam produzir impacto e benefícios a todos.

O ponto central deste quadro depende da informação, mídias e outros provedores de informação encontrados na internet e representa as fontes de informação que poderiam estar organizadas, arquivadas para uso e benefício de todos, via cloud computing. 
QUADRO 1. DESENVOLVIMENTO DE ESTRATÉGIAS, AGENTES, METAS E IMPACTOS

\begin{tabular}{|c|c|c|c|}
\hline $\begin{array}{c}\text { DIMENSÃO } \\
\text { CONTEXTUAL }\end{array}$ & ÁREAS DE ENFOQUE & METAS/ESTRATÉGIAS & IMPACTOS \\
\hline \multirow{2}{*}{$\begin{array}{l}\text { Sociedade do } \\
\text { Conhecimento }\end{array}$} & Educação Formal & \multirow{5}{*}{$\begin{array}{l}\text { Design Thinking para } \\
\text { criação de projetos } \\
\text { contextualizados } \\
\text {. Programas de formação } \\
\text { permanente } \\
\text {.Interação universidade x } \\
\text { espaços educacionais } \\
\text {. Programas extra } \\
\text { curriculares, parcerias e } \\
\text { conexões com programas } \\
\text { de educação formal, sites } \\
\text { desenvolvidos por } \\
\text { estudantes, festivais de } \\
\text { cinema, de games, } \\
\text { parcerias público-privadas } \\
\text { etc. }\end{array}$} & \multirow{5}{*}{$\begin{array}{l}\text { Desenvolvimento de } \\
\text { competências } \\
\text { midiáticas e } \\
\text { informacionais de } \\
\text { acesso a todos e de } \\
\text { mesma qualidade para } \\
\text { todos } \\
\text {. Uso de estratégias } \\
\text { pedagógicas, seleção } \\
\text { de recursos, } \\
\text { desenvolvimento e } \\
\text { implementação de } \\
\text { soluções } \\
\text { Formação de } \\
\text { professores } \\
\text { apropriados de cultura } \\
\text { digital e informacional }\end{array}$} \\
\hline & & & \\
\hline \multirow{8}{*}{$\begin{array}{l}\text { Cultura digital } \\
\text { Liberdade de } \\
\text { expressão e de } \\
\text { criação } \\
\text {. Pluralismo } \\
\text { Multimidiático } \\
\text {. Mídias } \\
\text { provedoras de } \\
\text { informação ética, } \\
\text { confiável e de } \\
\text { qualidade para } \\
\text { todos }\end{array}$} & $\begin{array}{l}\text { Conselhos Educacionais, } \\
\text { Diretorias de escolas, } \\
\text { universidades }\end{array}$ & & \\
\hline & $\begin{array}{l}\text { Espaços Educacionais } \\
\text { Informais }\end{array}$ & & \\
\hline & $\begin{array}{l}\text { Museus, Bibliotecas, } \\
\text { Associações profissionais, } \\
\text { ONGs, órgãos } \\
\text { responsáveis pela } \\
\text { produção e regulação das } \\
\text { mídias e de informações } \\
\text { etc. }\end{array}$ & & \\
\hline & Espaços Informais & & \\
\hline & $\begin{array}{l}\text { Diretores/produtores de } \\
\text { filmes, agência de } \\
\text { radiodifusão, Conselhos } \\
\text { de Publicidade, ONGs, } \\
\text { parques temáticos, } \\
\text { institutos }\end{array}$ & $\begin{array}{l}\text { programas na internet, } \\
\text { bibliotecas, museus, } \\
\text { institutos culturais, teatro, } \\
\text { mídia impressa e } \\
\text { radiodifusão, parcerias } \\
\text { com empresas etc. }\end{array}$ & $\begin{array}{l}\text {.Relevância da mídia, } \\
\text { internet e outros } \\
\text { provedores de } \\
\text { informação que } \\
\text { possam ser usados } \\
\text { para compartilhar } \\
\text { cultura, democracia, } \\
\text { paz, ética etc. }\end{array}$ \\
\hline & Mundo Corporativo & \multirow{3}{*}{$\begin{array}{l}\text { Elaborar mecanismos de } \\
\text { gerenciamento e de } \\
\text { avaliação; adoção da } \\
\text { sustentabilidade } \\
\text { educacional ou de outros } \\
\text { aspectos importantes; } \\
\text { formulação de pesquisas, } \\
\text { difusão de resultados. }\end{array}$} & \multirow{3}{*}{$\begin{array}{l}\text { Definição e avaliação } \\
\text { necessidades } \\
\text { informacionais, } \\
\text { utilizadas de maneira } \\
\text { ética, colaborativa e de } \\
\text { produção de conteúdo } \\
\text { contextualizado. }\end{array}$} \\
\hline & . Empresas de mídias, & & \\
\hline & $\begin{array}{l}\text { Associações de } \\
\text { jornalistas, } \\
\text { desenvolvedores de sites, } \\
\text { telecomunicação, } \\
\text { entretenimento etc. }\end{array}$ & & \\
\hline $\begin{array}{l}\text { Contexto } \\
\text { Educacional }\end{array}$ & $\begin{array}{l}\text {. Desenvolvimento da } \\
\text { sustentabilidade } \\
\text {. Reforma curricular para } \\
\text { uma educação c/ TIC } \\
\text {. Formação de } \\
\text { professores (Digital } \\
\text { Literacy; Informational } \\
\text { Literacy e estudos TPACK }\end{array}$ & $\begin{array}{l}\text { Promover parcerias, criar } \\
\text { intercâmbio da } \\
\text { sustentabilidade } \\
\text { educacional c/ fomento da } \\
\text { informática nos âmbitos } \\
\text { regionais, nacionais e } \\
\text { internacionais. }\end{array}$ & $\begin{array}{l}\text { Melhoria do acesso às } \\
\text { mesmas informações, } \\
\text { pesquisas, estudos, } \\
\text { aprendizagem e } \\
\text { demandas benéficas à } \\
\text { vida pessoal. }\end{array}$ \\
\hline Sustentabilidade & $\begin{array}{l}\text {.Transparência e } \\
\text { Responsabilidade } \\
\text {.Inclusão escolar e } \\
\text { inclusão social } \\
\text { Resiliência } \\
\text { Desenvolvimento } \\
\text { sustentável } \\
\text { Liberdade de associação, } \\
\text { tolerância, igualdade de } \\
\text { gêneros, fortalecimento } \\
\text { das pessoas etc. }\end{array}$ & $\begin{array}{l}\text { Manter provedores de } \\
\text { internet com competências } \\
\text { individuais, informação, } \\
\text { mudanças coletivas para o } \\
\text { bem comum; provedores } \\
\text { de memórias, } \\
\text { documentação de acordo } \\
\text { com suas } \\
\text { responsabilidades; } \\
\text { facilidade de acesso; } \\
\text { manter princípios } \\
\text { avançados da sociedade } \\
\text { de conhecimento etc. }\end{array}$ & $\begin{array}{l}\text {.Competências } \\
\text { individuais } \\
\text { transformadas em } \\
\text { mudanças coletivas } \\
\text { para o bem comum e } \\
\text { no desenvolvimento de } \\
\text { conquistas como o fácil } \\
\text { acesso às mídias, } \\
\text { instituições de } \\
\text { memória, de } \\
\text { documentação, } \\
\text { armazenadas conforme } \\
\text { princípio avançado da } \\
\text { sociedade do } \\
\text { conhecimento etc. }\end{array}$ \\
\hline
\end{tabular}

Fonte: Adaptado pelos autores The International Bank for Reconstruction and development; The World Bank, USA, 2005. 
As metas estratégicas sugeridas resumem algumas das ações que justificam o uso das informações e de engajamento, seja em espaços formais ou informais. $\mathrm{O}$ acesso, a função social de cada contexto, as estratégias adequadas podem favorecer a conscientização dos cidadãos sobre a finalidade da Sustentabilidade, de forma geral, assim como o conhecimento e análise crítica de suas necessidades informacionais.

Os impactos em cada contexto referem-se ao conhecimento básico que todos cidadãos devem ter sobre suas funções, natureza e padrões profissionais e éticos, estabelecidos ou não em cada mídia acessada.

Estas dimensões representam uma grande questão tanto para a Educação quanto para a Computação, ou seja, reunir ações multidisciplinares para criar plataformas distribuídas de auxílio à qualificação profissional e ao enriquecimento intelectual da população. Essas plataformas devem integrar ferramentas de comunicação, por exemplo, compatíveis com a Web como um enorme sistema operacional dinâmico e inteligente.

No caso da Educação Escolar significa: saber utilizar as estratégias da Pedagogia de Projetos, a partir de um problema formulado sobre temática interdisciplinar; prever, seja qual for a escolha da tecnologia a ser utilizada, as competências de explorar o que a escola possui, explorar as ideias existentes; prototipar modelos para resolução de problemas que envolvam raciocínio computacional (analisar, sintetizar, argumentar, investigar hipóteses); atuar em equipe; tomar decisões; saber argumentar com criatividade e inovação.

Pesquisas acadêmicas podem colaborar com a imersão nas escolas para realizar os desafios apontados com a finalidade de ampliar o desenvolvimento da sustentabilidade educacional e apropriação das tecnologias via cloud computing.

As diretrizes educacionais e políticas podem reduzir o engessamento e/ou isolamento dos componentes curriculares existentes na escola, "vindos de cima (diretorias de ensino, MEC etc)". Requer a participação interdisciplinar dos professores, caso contrário, os docentes permanecem desmotivados e pouco empenhados no desenvolvimento da educação com tecnologias.

Buscar formas inovadoras de avaliação podem eliminar a questão da competitividade; da punição pelo erro. A avaliação de competências e habilidades previstas pela legislação educacional precisa ter caráter processual com atividades dinâmicas e flexíveis, não se esquecendo das atividades de recuperação que podem ser fortemente apoiadas pelas tecnologias digitais.

Outra estratégia que merece destaque é saber fazer escolhas metodológicas contextuais, mais próximas das realidades dos alunos, com ênfase na autonomia e nos pressupostos da gamificação, sem gestão do tipo tradicional de comando-controlesupervisão; dar relevância aos grupos focais para que os feedbacks possam ser ouvidos, com mecanismos de autoavaliação, vivência compartilhadas das hipóteses construtivas (e não dos "erros").

\section{Implicações Finais}

Este artigo estabeleceu algumas provocações para ampliar o debate e os estudos sobre o tema, ainda restrito para a realidade escolar brasileira, de qualquer nível ou modalidade de ensino. Diante delas, o que se pode pensar sobre o futuro é como tornar a educação sustentável com os recursos que cada espaço de ensino e de aprendizagem possuem e como a computação pode auxiliar. 
O artigo defende que a comunidade mediada pelas tecnologias não pode ser estudada apenas pelos avanços tecnológicos ou comerciais de sua oferta, mas pelo impacto sociocultural que produzem nas pessoas, em seus contextos de vida e de relações interpessoais. Critica a subutilização das potencialidades interativas da web, suas dificuldades de acesso, a heterogeneidade na construção das identidades e defende nova postura comunicacional, alicerçada na autoria do usuário, na lógica da interatividade e constantemente (re) significada.

\section{Referências}

Barr, V. e Stephenson, C. (2011). Bringing computational thinking to K-12: what is Involved and what is the role of the computer science education community?. Acm Inroads, 2(1), 48-54.

Blikstein, P. (2008). "O Pensamento Computacional e a Reinvenção do Computador na Educação”. Disponível em: < http://bit.ly/11XlbNn>. Acesso em: 10 de abr. 2016.

Blikstein, P. (2010 a). Can non-intelligent behavior generate intelligence? Multi-agent computational modeling as a theory-building tool in developmental psychology and education research. Jean Piaget Society Annual Conference (JPS 2010), Saint Louis, MO.

Blikstein, P. (2010 b) [symposium organizer and chair]. A New Age in Digital Tangible Interfaces for Learning. In K. Gomez, L. Lyons, \& J. Radinsky (Eds.), Learning in the Disciplines: Proceedings of the 9th International Conference of the Learning Sciences (ICLS 2010). University of Illinois at Chicago: Chicago, IL.

Pereira, A. Bosco e Piconez, S C B. (2016) Abordagem Interativa na aprendizagem da Matemática com uso de games no celular e os conteúdos curriculares do Ensino Fundamental. Anais do SBIE, Uberlândia, 2016.

Brennan, K. e Resnick, M. (2012). New frameworks for studying and assessing the development of computational thinking. In Proceedings of the 2012 annual meeting of the American Educational Research Association, Vancouver, Canada.

Cassel, L. N. (2011). Interdisciplinary computing is the answer: now, what was the question?. ACM Inroads, vol. 2, issue 1. p. 46.

De Paula, B. H., Valente, J. A. e Burn, A. (2014). O uso de jogos digitais para o desenvolvimento do currículo para a Educação Computacional na Inglaterra. Currículo sem Fronteiras, v. 14, n. 3, p. 46-71.

Ekanayake, Jaliya and Fox, Geoffrey. (2010) "High Performance Parallel Computing with Clouds and Cloud Technologies", in Lecture Notes of the Institute for Computer Sciences, Social Informatics and Telecommunications Engineering. Volume 34, 2010, pp 20-38.

Grassian, E.S.; Kaplpwitz, J.R. (2001) Information Literacy Instruction: theory and practice. Edison, NJ: Neal-Schumam Publishers.

Mestre, P. et al. (2015) Pensamento Computacional: Um estudo empírico sobre as questões de matemática do PISA. In: Anais dos Workshops do Congresso Brasileiro de Informática na Educação. p. 1281

NIST (2013) National Institute for Standards and Technology of The NIST Definition of Cloud Computing (NIST Special Publication 800-145). Disponível em: http://csrc.nist.gov 
Piconez, Stela. C B. (2004) Sistema Transversal de Ensino-Aprendizagem: por uma pedagogia de inclusão escolar. Cadernos Pedagógicos Reflexões (USP), São Paulo, v. 1, n. 4, p. $9-50$.

Piconez, S C B et al. (2013) "Formação Permanente de Educadores, Recursos Educacionais Abertos e Integração dos Conhecimentos". In: Recursos Educacionais Abertos e redes sociais. Okada, A. (org.) 2a. ed. São Luís,MA.

Righi, R. da Rosa. (2013) Elasticidade em cloud computing: conceito, estado da arte e novos desafios, In Revista Brasileira de Computação Aplicada, v.5, n2., São Paulo.

THE INTERNATIONAL BANK FOR RECONSTRUCTION AND DEVELOPMENT: THE WORLD BANK. (2005) Conceptual Framework for ICT in Monitoring and Evaluation of ICT in Educational projects, USA.

Tran V, et al. (2013) On the underlying assumptions of threshold Boolean networks as a model for genetic regulatory network behavior. Front Genet 4:263

Zufo et al. (2013). A Computação em nuvem na Universidade de São Paulo. In Revista da USP, no.97.

Zanetti, H.A.P. et al. (2016) Pensamento Computacional no Ensino de programação: uma revisão sistemática da literatura brasileira. In Anais do XXVII Simpósio Brasileiro de Informática na Educação. 